exposure route (85\%). Biological response to sarin exposure was expressed as percentage change in cholinesterase activity and, less commonly, change in pupil size. For inhalation tests, assessed by changes in red blood cell cholinesterase median inhibition for was 41\% (IQR 24\%-51\%), with a maximum of $87 \%$. For dermal exposures, assessed by changes in unspecified cholinesterase, median inhibition was 22\% (IQR 10\%$37 \%$ ), with a maximum inhibition of $99 \%$. There was a clear association between increasing exposure to sarin and depression of cholinesterase activity but the strength $f$ the association varied by exposure route and the presence of chemical or physical protection. Pupil size decreased with increased exposure but this relationship was less apparent when protection was present.

Conclusions These results, drawn from high quality experimental data, offer a unique insight into the effects of these chemical agents on humans.

Acknowledgement We thank Nicola Fear and Gemma Archer, our collaborators in new work on these data, for their input.

\section{C.6 THE HEALTH AND WELLBEING OF NEW ZEALAND VETERANS}

${ }^{1}$ David McBride*, ${ }^{2}$ Dianne Gardner, ${ }^{1}$ Amy Richardson, ${ }^{1}$ Emma Wyeth, ${ }^{1}$ Ari Samaranayaka, ${ }^{1}$ Gagan Gurung, 'Sarah Derrett. 'University of Otage, Dunedin, New Zealand; 'Massey University, Palmerston North, New Zealand

\subsection{6/OEM-2019-EPI.127}

The psychological and physical health and wellbeing of New Zealand contemporary veterans

Background For New Zealand veterans, operational service and transition to civilian life are critical life events. Most veterans do well, but a significant minority fare poorly. Adverse outcomes are associated with post-traumatic stress disorder, PTSD, and also the development of minor but multiple health complaints, 'multiple symptom illness' (MSI).

Aim This project investigates factors associated with both wellbeing and ill health in NZ veterans, with the aim of developing a parsimonious instrument to detect 'at risk' veterans prior to, or at, transition.

Methods An on-line cross-sectional survey.

Wellbeing is measured by the EQ-5D, a health-related quality of life instrument assessing mobility, self-care, usual activities, pain/discomfort, and anxiety/depression. The adverse outcome is PTSD, identified using the PCL-M, an instrument reflecting DSM-IV PTSD symptoms.

Risk factors include MSI conforming to the Centers for Disease Control definition as 'the presence, for at least six months, of one or more chronic symptoms from at least two of three categories, namely fatigue, mood-cognition and musculoskeletal symptoms'. The General Health Questionnaire-28 (GHQ-28) screens for emotional distress and possible psychiatric morbidity. The Brief Trauma Questionnaire (BTQ), a 10 -item self-report questionnaire derived from the Brief Trauma Interview, assesses the role of stressors contributing to PTSD.

Protective factors include the 24-item Social Provisions Scale (SPS) assessing social relationships and various dimensions of social support.

Analysis Logistic regression will identify the model of best fit for PTSD (PCL-M) and wellbeing (EQ-5D) respectively.
For MSI, a factor analysis will describe the pattern of symptom reporting. If this matches international experience, 3 factors will explain the majority of the variance in the data. Results As at the 14th Nov 2018 we have 1592 completed questionnaires. Analysis will commence in mid-November 2018.

\section{Exposure Assessment-2}

\section{D.1 ABSTRACT WITHDRAWN}

\section{D.2 OCCUPATIONAL EXPOSURE OF HEALTHCARE PERSONNEL TO NITROUS OXIDE IN VARIOUS PEDIATRIC SPECIALTY CARE UNITS: AN OBSERVATIONAL STUDY}

\begin{abstract}
${ }^{1,2}$ Marie-Agnès Denis* ${ }^{3}$ Charlotte Pete-Bonneton, ${ }^{4,5}$ Benjamin Riche, ${ }^{6}$ Robert Cadot, ${ }^{1,3}$ Amélie Massardier-Pilonchery, ${ }^{4,5}$ Jean Iwaz, ${ }^{1,3}$ Barbara Charbotel. ${ }^{1}$ Univ Lyon, Université Claude Bernard Lyon1, Ifsttar, Umrestte, Umr T_9405, F- 69373, Lyon; '2Service de médecine et santé au travail, Hospices Civils de Lyon - 59 Bd Pinel, F- 69677 Bron cedex; ${ }^{3}$ Service des Maladies Professionnelles, Centre Hospitalier Lyon Sud, Hospices Civils de Lyon, Pierre Bénite, France; ${ }^{4}$ Service de Biostatistique - Bioinformatique, Pôle Santé Publique, Hospices Civils de Lyon, Lyon, France; ${ }^{5}$ Univ Lyon, Université Claude Bernard Lyon 1, CNRS UMR 5558, Laboratoire de Biométrie et Biologie Évolutive, Équipe Biostatistique-Santé, Villeurbanne, France; ${ }^{6}$ Laboratoire de Toxicologie Professionnelle et Environnementale, Centre Hospitalier Lyon Sud, Hospices Civils de Lyon, Pierre Bénite, France
\end{abstract}

\subsection{6/OEM-2019-EPI.128}

Objectives Nitrous oxide (N2O) present in anesthetic mixtures (e.g., equimolar mix of oxygen and N2O, EMONO) has been found responsible for various toxicities, including genetic and reproductive toxicities. N2O-containing mixtures are widely used in pediatric care units where most healthcare providers (HCPs) are women of childbearing age. This motivated an investigation of occupational exposure to $\mathrm{N} 2 \mathrm{O}$ in search for overexposure and overexposure factors in a pediatric hospital.

Methods This observational study concerned seven different units. On each of 34 HCPs, air samples were extracted by portable pumps and collected in Tedlar ${ }^{\circledR}$ bags. N2O was quantified by gas chromatography coupled to pulsed discharge ionization detection and infrared spectrometry. The data allowed calculating mainly the instantaneous exposure and the 8 hour time-weighted average (8h-TWA).

Results The exposure was four times higher in closed than in open treatment rooms and two times higher in case of use vs. non-use of EMONO. The exposure was significantly higher in junior vs. senior HCPs (by 12\%) and higher during presumably short vs. presumably long procedures (by 20\%). The mean $8 \mathrm{~h}-\mathrm{TW}$ As were rather higher than the recommended exposure limit (25 ppm/8 hour) in emergency unit and in day hospital for thoracic and abdominal diseases. Overexposures represented $11 \%$ of all measurements but reached substantial levels (up to 3.5 times the recommended threshold).

Conclusions Overexposures to $\mathrm{N} 2 \mathrm{O}$ were frequent during short-duration procedures. The causes of overexposure were insufficient air renewal and inappropriate equipment use. This calls for dedicated rooms, more efficient medical/nursing practices, proper training, and regular checks of gas levels. 